\title{
Linfoma no Hodgkin de Mama en el Instituto Nacional de Oncología de Cuba, en el período comprendido entre 1980 y 2010.
}

*Correspondencia:

julio.jimenez@infomed.sld.cu Teléfono [53] 78382578

Conflicto de intereses: Los autores declaran no tener conflictos de intereses.

Fondos: Ver la página 235

Recibido: 1 Agosto 2016

Aceptado: 19 Marzo 2017

Publicado: 30 Diciembre 2017

Membrete bibliográfico:

Franco S, Jiménez J, Delgado R, López L. Linfoma no Hodgkin de Mama en el Instituto Nacional de Oncología de Cuba, en el período comprendido entre 1980 y 2010. Rev. Oncol. Ecu 2017;27(3):228237.

DOI: https://doi.org/10.33821/066

Copyright Franco, et al. Este artículo es distribuido bajo los términos de Creative Commons Attribution License, el cual permite el uso y redistribución citando la fuente y al autor original.

\section{Breast non-Hodgkin Iymphoma at the National Institute of Oncology of Cuba, in the period between 1980 and 2010.}

\section{Sonia Olga Franco Odio1* ${ }^{*}$, Julio Jesús Jiménez Galainena1 ${ }^{\text {ID, }}$ Rodolfo Delgado Almanza1, Leonel P. López Rodríguez.}

1. Instituto Nacional de Oncología y Radiobiología, La Habana-Cuba.

\section{Resumen}

Introducción: Los Síndromes Linfoproliferativos son procesos neoplásicos que se originan en el tejido linfoide tanto central como periférico. La presentación de Linfomas no Hodgkin de mama es infrecuente, por lo que se presenta una serie de casos de esta patología en un centro oncológico nacional en la Habana-Cuba.

Métodos: El presente estudio observacional, retrospectivo fue realizado en el Instituto Nacional de Oncología de Cuba. Se revisaron historias clínicas y registros de anatomía patológica de pacientes con cáncer de mama del Instituto Nacional de Oncología y Radiobiología de Cuba, de los períodos comprendidos de enero de 1980 a diciembre de 2010. El tipo de muestreo fue no probabilístico de conveniencia. Las variables estudiadas fuero sexo, edad, diagnóstico anatomopatológico, localización de las lesiones y supervivencia. Se utiliza estadística descriptiva para datos descriptivos y Kaplan Meier para el reporte de supervivencia.

Resultados: Se analizaron 14 registros de pacientes mujeres con edad media de $54.7 \pm 18$ años. La duración de los síntomas promedio de $9.2 \pm 18$ meses. 13 casos fueron localizados en la mama derecha (92.86 \%). En diez casos (71.53\%) se presentaron con un estadio clínico I. El diagnóstico histopatológico más prevalente fue el de Linfoma No Hodgkin (LNH) difuso de células grandes B con 3 casos (21.4\%) y LNH difuso mixto de células pequeñas y grandes hendidas con 3 casos (21.4\%). Trece de los casos (92.86 \%) fueron sometidos a cirugía de mastectomía, 13 casos (92.86 \%) fueron sometidos a Quimioterapia y en 6 casos (42.86 \%) se aplicaron sesiones de radioterapia. La supervivencia del grupo de pacientes fue reportada en $74 \pm 16.5$ meses, (IC 95\% 41.6 - 106.4).

Conclusión: En esta serie de casos presentada la incidencia de Linfoma No Hodgkin de mama fue de 14 casos, el gran porcentaje en mama derecha, la supervivencia del grupo de pacientes fue de 70 
meses. La histología prevalente fue de células B y Linfoma No Hodgkin difuso mixto de células pequeñas y grandes hendidas.

Palabras Claves: NEOPLASIAS DE LA MAMA, LINFOMA NO HODGKIN, SUPERVIVENCIA (SALUD PÚBLICA).

DOI: $10.33821 / 066$

\section{Abstract}

Introduction: Lymphoproliferative Syndromes are neoplastic processes that originate in the lymphoid tissue, both central and peripheral. The presentation of non-Hodgkin lymphomas of the breast is infrequent, which is why a series of cases of this pathology is presented at a national cancer center in Havana-Cuba.

Methods: The present observational, retrospective study was conducted at the National Institute of Oncology of Cuba. Clinical histories and pathological anatomy records of patients with breast cancer were reviewed from the National Institute of Oncology and Radiobiology of Cuba, from the periods of January 1980 to December 2010. The type of sampling was non-probabilistic of convenience. The variables studied were sex, age, anatomopathological diagnosis, location of the lesions and survival. Descriptive statistics are used for descriptive data and Kaplan Meier for the survival report.

Results: We analyzed 14 records of female patients with an average age of $54.7 \pm 18$ years. The average symptom duration was $9.2 \pm 18$ months. 13 cases were located in the right breast $(92.86 \%)$. In ten cases $(71.53 \%)$ they presented with a clinical stage I. The most prevalent histopathological diagnosis was that of diffuse large non-Hodgkin lymphoma (NHL) of B cells with 3 cases $(21.4 \%)$ and mixed diffuse NHL of small and large cells cleft with 3 cases $(21.4 \%)$. Thirteen of the cases $(92.86 \%)$ underwent mastectomy surgery, 13 cases $(92.86 \%)$ underwent chemotherapy and in 6 cases $(42.86 \%)$ radiotherapy sessions were applied. The survival of the group of patients was reported in $74 \pm 16.5$ months, (95\% Cl 41.6 - 106.4).

Conclusion: In this case series presented the incidence of non-Hodgkin's lymphoma of the breast was 14 cases, the large percentage in the right breast, the survival of the group of patients was 70 months. The prevalent histology was B cells and mixed diffuse non-Hodgkin lymphoma of small and large cells cleaved.

Keywords: BREAST NEOPLASMS, NON-HODGKIN LYMPHOMA, SURVIVAL (PUBLIC HEALTH).

DOI: $10.33821 / 066$

\section{Introducción}

Los Síndromes Linfoproliferativos (SL) son procesos neoplásicos que se originan en el tejido linfoide tanto central como periférico, presentando diversas variedades morfológicas, inmunológicas, genéticas y clínicas, con expresión de la variedad citológica y de la diversidad de la función inmune que corresponde a estas estructuras [1].

La clasificación WHO 2008 agrupa los procesos linfoproliferativos como neoplasias de células $B$, neoplasias de células $T$ y linfoma de Hodgkin [2]. Aunque desde el punto de vista 
clínico corresponden los linfomas No Hodgkin B o T, la enfermedad de Hodgkin y las leucemias linfoides agudas y crónicas; se ha establecido que corresponden a Linfomas $B$ $85 \%$, a Linfomas T el $15 \%$, mientras que el $25 \%$ de ellos son Linfomas Extraganglionares. La localización más frecuente es en el tracto gastrointestinal, seguido por el anillo de Waldeyer. La mama abarca el $2 \%$ de los linfomas extranodales [3].

La mayoría de los autores han encontrado que los Linfomas difusos de células grandes $B$ es la variedad más frecuente, representada por el $31 \%$, el Linfoma Folicular con el $22 \%$ en segundo lugar y en tercer lugar el Linfoma marginal o MALT con el $8 \%$ [4].

Brogi y Lee Harris plantean que la mama derecha está afectada con mayor frecuencia y es bilateral sincrónico entre el 5 y $25 \%$. Vikas encontró el $13 \%$ bilateral sincrónico y el $7 \%$ metacrónico $[5,6]$.

Desde el punto de vista de los estudios mamográficos, aparecen como nódulos únicos o múltiples, sin calcificaciones, de 2 a $5 \mathrm{~cm}$, con bordes lisos, lobulados o parcialmente espiculados o un incremento difuso y heterogéneo de la densidad mamaria. Ecográficamente como nódulos sólidos, hipoecogénicos, heterogéneos con bordes lisos o digitiformes, con márgenes bien definidos y refuerzo acústico posterior.

La estadificación de Ann Arbor y la edad son los dos factores pronósticos más relevantes y de ellos, es la estadificación el de mayor significación estadística.

El uso multidisciplinario de técnicas de anatomía patológica puede eliminar la necesidad de procederes quirúrgicos. Por ejemplo si se le realiza aspiración con aguja fina y el extendido obtenido está constituido por una población de células discohesivas de gran y pequeño tamaño podemos realizar Inmunocitoquímica y diagnosticar un Linfoma No Hodgkin Mixto Difuso. Aunque la biopsia aspiración con aguja fina (BAAF) en el linfoma de mama es un procedimiento fácil a menudo a través de ésta es imposible diferenciar un linfoma de bajo Grado de una proliferación reactiva, a no ser que se use marcadores linfoides para antígenos B y T, cadenas ligeras Kappa y Lambda, Bcl-2, Ciclina D1, Ki-67 entre otros [7]. Debido a que la incidencia de Linfoma no Hodgkin de mama es muy baja se presenta una serie de casos en un centro oncológico único en la Habana-Cuba de un período de análisis de 30 años y se reporta la supervivencia de este grupo.

\section{Materiales y Métodos}

Se realiza un estudio retrospectivo y observacional en el período comprendido de enero de 1980 y diciembre de 2010 de los registros del Departamento de Anatomía Patológica del Instituto Nacional de Oncología y Radiobiología de Cuba. Ingresaron al estudio los registros de pacientes con diagnósticos de Linfomas malignos en la mama.

El tipo de muestreo fue no probabilístico de conveniencia, del período estudiado. Se excluyeron casos en los que no se tuvo la información clínica completa. Los diagnósticos provenían de biopsias realizadas por punción con aguja fina o de estudios patológicos de nódulos de mama cuyas imágenes resultaron sospechosas de malignidad para los 
radiólogos y oncólogos. Las piezas quirúrgicas se describieron y se tomaron fragmentos para procesarlos en el Laboratorio de Histopatología.

Se emplearon tinciones de Hematoxilina y Eosina, posteriormente Inmunotinciones con diversos anticuerpos de acuerdo a los protocolos establecidos previamente con el objetivo de conocer la estirpe celular.

Estos datos fueron ingresados en una matriz del software de análisis estadístico IBM SPSS 22. Las variables estudiadas fuero sexo, edad, diagnóstico anatomopatológico, localización de las lesiones y supervivencia. Se utiliza estadística descriptiva para las variables descriptivas. Para la supervivencia se utiliza Kaplan Meier.

\section{Resultados}

Ingresaron al estudio 14 casos de mujeres con Linfoma No Hodgkin de mama. La edad promedio del grupo de estudio fue de $54.7 \pm 18$ años. La duración de los síntomas fue en promedio $9.2 \pm 18$ meses. 13 casos fueron localizados en la mama derecha (92.86 \%). En diez casos $(71.53 \%)$ se presentaron con un estadio clínico I (Tabla 1).

Tabla 1. Datos clínicos de los pacientes con Linfoma No Hodgkin de mama.

\begin{tabular}{|l|c|c|c|c|}
\hline Casos & Edad & $\begin{array}{c}\text { Duración } \\
\text { síntomas } \\
\text { (meses) }\end{array}$ & Localización & Estadio \\
\hline $\mathbf{1}$ & 42 & 3 & $\begin{array}{c}\text { MD + MI + Pared } \\
\text { abdominal }\end{array}$ & IV EB \\
\hline $\mathbf{2}$ & 43 & 72 & MD & IV EB \\
\hline $\mathbf{3}$ & 79 & 3 & MD & I \\
\hline $\mathbf{4}$ & 46 & 2 & MD & I EA \\
\hline $\mathbf{5}$ & 47 & 4 & MD & IV \\
\hline 6 & 21 & 2 & MI & I \\
\hline 7 & 31 & 12 & MD & I \\
\hline $\mathbf{8}$ & 50 & 1 & MD & I \\
\hline $\mathbf{9}$ & 78 & 3 & MD & I \\
\hline 10 & 64 & 4 & MD & I \\
\hline 11 & 58 & 5 & MD & I \\
\hline 12 & 65 & 6 & & \\
\hline 13 & 77 & 4 & & \\
\hline 14 & 65 & 8 & & \\
\hline
\end{tabular}

MD: Mama Derecha. MI: Mama Izquierda.

El diagnóstico histopatológico más prevalente fue el de Linfoma No Hodgkin difuso de células grandes B con 3 casos (21.4\%) y LNH difuso mixto de células pequeñas y grandes hendidas con 3 casos (21.4\%) (Tabla 2). 
Tabla 2. Diagnóstico histopatológico de la serie de casos.

\begin{tabular}{|l|c|c|}
\hline Diagnóstico Histopatológico & $\mathbf{n = 1 4}$ & Porcentaje 100\% \\
\hline LNH difuso de células grandes B & 3 & $21.43 \%$ \\
\hline $\begin{array}{l}\text { LNH difuso mixto de células pequeñas y grandes } \\
\text { hendidas }\end{array}$ & 3 & $21.43 \%$ \\
\hline LNH difuso de células pequeñas & 2 & $14.29 \%$ \\
\hline Linfoma difuso de células pequeñas hendidas & 1 & $7.14 \%$ \\
\hline LNH & 1 & $7.14 \%$ \\
\hline LNH difuso de células grandes B Subtipo anaplásico & 1 & $7.14 \%$ \\
\hline LNH difuso de células pequeñas hendidas & 1 & $7.14 \%$ \\
\hline LNH folicular y difuso de pequeñas células & 1 & $7.14 \%$ \\
\hline LNH linfohistiocítico & 1 & $7.14 \%$ \\
\hline
\end{tabular}

LNH: Linfoma No Hodgkin.

Trece de los casos (92.86 \%) fueron sometidos a cirugía de mastectomía, 13 casos (92.86 \%) fueron sometidos a Quimioterapia y en 6 casos (42.86 \%) se aplicaron sesiones de radioterapia (Tabla 3). Los datos de inmunohistoquímica reportaron positivos para CD20 en 5 casos $(35.71 \%)$, los mismos que fueron negativos para CD30 (Tabla 3).

Tabla 3. Diagnóstico histopatológico de la serie de casos.

\begin{tabular}{|l|l|l|l|c|c|}
\hline Casos & $\begin{array}{l}\text { Segui } \\
\text { miento } \\
(\text { Meses })\end{array}$ & Tipo & Tratamiento & CD 20 & $\begin{array}{l}\text { CD } \\
\mathbf{3 0}\end{array}$ \\
\hline $\mathbf{1}$ & 36 & LNH DCG-B & Cir + QTP + RTP & + & - \\
\hline $\mathbf{2}$ & 204 & LNH linfohistiocítico & Cir + QTP + RTP & & \\
\hline $\mathbf{3}$ & 48 & LNH & Cir & & \\
\hline $\mathbf{4}$ & 36 & LNH DCG B, Subtipo anaplásico & Cir + QTP + RTP & + & - \\
\hline $\mathbf{5}$ & 156 & LNH DCP y DCG hendidas & QTP + RPT & & \\
\hline $\mathbf{6}$ & 132 & LNH DCP y DCG hendidas & Cir + QPT & & \\
\hline $\mathbf{7}$ & 132 & LNH DCP hendidas & Cir + QTP + RTP & & \\
\hline $\mathbf{8}$ & 84 & LNH DCG-B & Cir + QTP + RTP & + & - \\
\hline $\mathbf{9}$ & 60 & LNH DCP hendidas & Cir + QTP & & \\
\hline $\mathbf{1 0}$ & 12 & LNH DCP y DCG hendidas & Cir + QTP & & \\
\hline $\mathbf{1 1}$ & 24 & LNH DCG-B & Cir + QTP & + & - \\
\hline $\mathbf{1 2}$ & 96 & LNH folicular y DCP & Cir + QTP & & \\
\hline $\mathbf{1 3}$ & 12 & LNH DCP & Cir + QTP & & \\
\hline $\mathbf{1 4}$ & 4 & LNH DCP & Cir + QTP & + & - \\
\hline
\end{tabular}

LNH: Linfoma no Hodgkin. DCG: Difuso de células grandes. DCP: Difuso de células pequeñas. Cir: Cirugía. QTP: Quimioterapia. RTP: Radioterapia.

La supervivencia del grupo de pacientes fue reportada en $74 \pm 16.5$ meses, (IC 95\% 41.6 106.4) (Figura 1). 
Figura 1. Función de Supervivencia de Kaplan Meier de los pacientes con Linfoma no Hodgkin de mama.

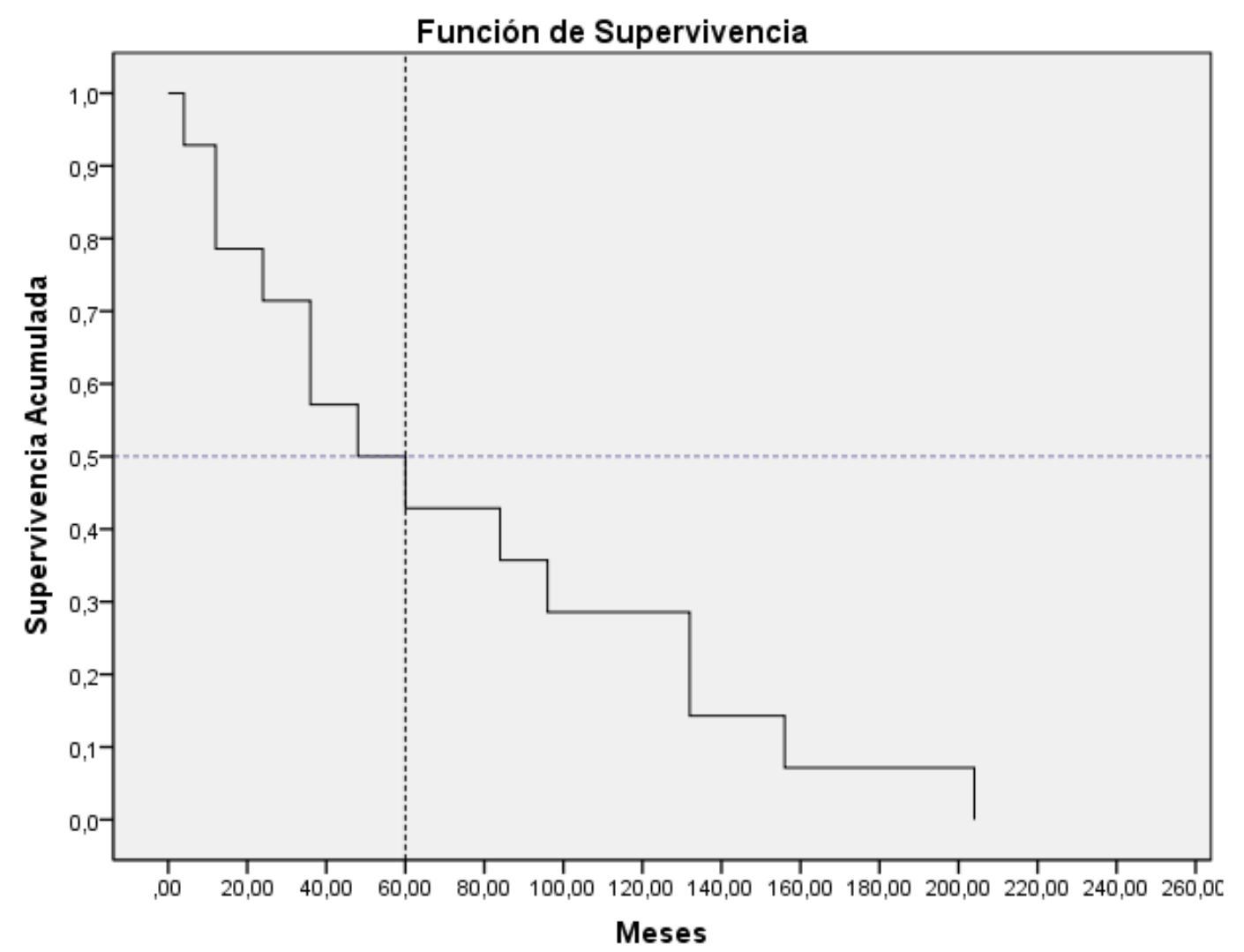

\section{Discusión}

En el presente estudio se presenta una serie de casos de Linfoma No Hodgkin de mama en 14 mujeres, con un prevalente porcentaje de afección en Mama Derecha, cifra semejante en estudios previos reportados [9]. El comienzo de los síntomas ha sido variable. Todos los casos mostraron estudios imagenológicos con criterios de malignidad y se valoraron como posibles carcinomas de mama. Se encontraron en etapas tempranas de la enfermedad y con el tratamiento combinado de cirugía y quimioterapia, la supervivencia y el intervalo libre de enfermedad fueron largos $[10,11]$. Este estudio reporta la supervivencia de los pacientes de 74 meses con un intervalo de confianza del 95\% de 41.6 a 106.4 meses. El estudio histopatológico intraoperatorio fue diagnosticado por dos patólogos como procesos linfoproliferativos y se corroboró en las parafinas en todos los casos. En la presente serie de casos retrospectiva hay diferencias en los diagnósticos ya que se aplicaron en cada etapa las clasificaciones vigentes y no todos tienen inmunotinciones para definir la estirpe celular que compone la proliferación. La variedad más frecuente encontrada fue el Linfoma No Hodgkin difuso de células grandes $B$, lo que coincide con reportes previos [14, 15]. Las técnicas de Inmunohistoquímica ofrecen relevante información acerca del tipo celular y 
ayudan a emplear el esquema terapéutico más adecuado incluso con anticuerpos específicos [16].

Los beneficios de realizar o no mastectomías o exéresis amplias a estas pacientes han sido objeto de discusión entre los autores consultados y hay acuerdo en considerar que el pronóstico ha dependido del estado de los ganglios linfáticos extirpados. Además, el diagnóstico de la variedad puede ser obtenido en cualquier cantidad de tejido extraído sin llegar a mastectomías, ya que a veces hay varias lesiones en la misma mama, o en ambas y en otras localizaciones secundarias al tumor primario de la mama [17]. El Linfoma maligno primario de la mama constituye alrededor del $2 \%$ de las localizaciones extranodales y es más afectada la mama derecha en la serie presentada y en otras revisadas. Predominan los Linfomas No Hodgkin de células B corroborados con inmunotinciones con diversos marcadores que permiten conocer la estirpe celular.

Próximos estudios prospectivos deberán realizarse en donde se debe medir la supervivencia de grupos de pacientes con Linfoma No Hodgkin de mama de células B, células pequeñas y grupos mixtos.

\section{Conclusiones}

En esta serie de casos presentada la incidencia de Linfoma No Hodgkin de mama fue de 14 casos, el gran porcentaje en mama derecha, la supervivencia del grupo de pacientes fue de 70 meses. La histología prevalente fue de células B y Linfoma No Hodgkin difuso mixto de células pequeñas y grandes hendidas.

\section{Agradecimientos}

Reconocemos a las personas que participaron indirectamente en el estudio tales como el personal técnico, personal del departamento de anatomía patológica del Instituto Nacional de Oncología y Radiobiología, La Habana-Cuba.

\section{Información adicional}

\section{Nota del Editor}

La Revista Oncología Ecu permanece neutral con respecto a los reclamos jurisdiccionales en mapas publicados y afiliaciones institucionales.

\section{Abreviaturas}

LNH: Linfoma no Hodgkin.

DCG: Difuso de células grandes.

DCP: Difuso de células pequeñas. Cir: Cirugía.

QTP: Quimioterapia.

RTP: Radioterapia. 
Archivos Adicionales

Ninguno declarado por los autores.

Fondos

Los fondos de la investigación fueron propios de los autores del presente artículo.

\section{Disponibilidad de datos y materiales}

Existe la disponibilidad de datos bajo solicitud al autor de correspondencia. No se reportan otros materiales.

\section{Contribuciones de los autores}

SOFO, JJJG, realizaron la idea de investigación, revisión bibliográfica. RDA, LPLR recolección de datos, escritura del artículo, análisis crítico del artículo. JJJG realizó las correcciones editoriales. Todos los autores leyeron y aprobaron la versión final del artículo.

Aprobación de ética y consentimiento para participar

No aplica a este estudio.

\section{Consentimiento para publicación}

No aplica.

\section{Información de los autores}

Sonia Olga Franco Odio, Especialista de Segundo Grado en Anatomía Patológica. Instituto Nacional de Oncología y Radiobiología, La Habana-Cuba. +.

Julio Jiménez Galidena, Especialista Segundo Grado en Anatomia Patológica. Investigador Agregado. Profesor Instructor de la Universidad de Ciencias Médicas de La Habana. Instituto Nacional de Oncología y Radiobiología. La Habana, Cuba.julio.jimenez@infomed.sld.cu

Rodolfo Delgado Almanza, Especialista de Primer Grado en Oncología. Hospital Universitario Julio Trigo López. 
Leonel P. López Rodríguez, Especialista de Primer Grado en Oncología. Hospital Universitario Julio Trigo López.

\section{Revisiones por pares}

Acceda a la revisión de pares académicos en el siguiente enlace: https://publons.com/review/4048088

\section{Referencias}

Abreviaturas en la referencias

DOI: Digital Object

Identifier

PMID: PubMed Identifier

SU: Short URL
1. Jaffe E, Harris N, Stein H, Vardiman JW, editors. WHO Classification of Tumours. Pathology and Genetics of Tumours of the Haematopoietic and Lymphoid Tissue. Lyon: IARC Press, 2001.

2. Swerdlow SH, Campo E, Harris NL, Jaffe ES, Pileri SA, Stein H, et al. editors WHO Classification of Tumours of Haemopoietic and LymphoidTissues. Lyon: IARC Press, 2008.

3. Lamovec J, Wotherspoon A, Jacquemier J. Malignant lymphoma and metastatic tumours. In: Tavassoli F, Devilee $P$ (eds) Tumors of the breast and female genital organs. World Health Organization classification of tumours. IARC, Lyon, 2003:107-109.

4. Isaacson PG, Norton AJ. Mucosa-associated lymphoid tissue and the MALT lymphoma concept. En: Extranodal lymphomas. Edimburg: Churchill livingston, 1994:5-14.

5. Brogi E, Harris NL. Lymphomas of the breast: pathology and clinical behavior. Semin Oncol 1999; 26:357-364.

6. Talwalkar SS, Miranda RN, Valbuena JR, Routbort MJ, Martin AW, Medeiros LJ. Lymphomas involving the breast: a study of 106 cases comparing localized and disseminated neoplasms. Am J Surg Pathol 2008;32:1299-1309.

7. Balague $\mathrm{O}$, Ott G, Hasserjian R, Elenitoba-Johnson K, de Leval L, de Jong D. Commentary on the WHO classification of tumors of lymphoid tissues (2008): aggressive B-cell lymphomas JHematop. 2009;2(2):83-87.

8. Matsushima AY, Hamele-Bona D, Osborne BM. Fine-needle aspiration biopsy findings in marginal zone B cel lymphoma. Diagn Cytopathol 1999;20(4):190-8.

9. Vianello F, Sartori D, Girolami A. Primary breast lymphoma. Forum (Genova) 1998;8(2):188-95.

10. Lee KC, Chang HT, Chen CJ, Mok KT, Chung hua I, Hsuech Tsa. Primary non-Hodgkin's lymphoma of the breast conservation therapy in two patients. Chih (Taipei) 1999;62(9):633-8.

11. Michetti M, Minelli M, Berni C, Bellini V, La Porta A, Manente L, et al. A case of primary non-Hodgkin lymphoma of the breast. Clin Ter 1999;150(4):307-10.

12. Gholam D, Bibeau F, El Weshi A, Bosq J, Ribrag V. Primary breast lymphoma. Leuk Lymphoma 2003;44:1173-1178.

13. Farkash E, Ferry J, Lee Harris N, Hochberg E, Takvorian R, Zuckerman D, Sohani A. Case report Rare lymphoid malignancies of the breast: a report of two cases illustrating potential diagnostic pitfalls $\mathrm{J}$ Hematop. 2009:2(4):237-244. DOI: 10.1007/s12308-009-0043-y

14. Hugh JC, Jackson FI, Hauson J, Poppema S. primary breast lymphoma au immunohistologic study of 20 new cases. Cancer 1990; 66(12):2602-11. 
15. Wang XQ, Zhang LX, Bi CF, Luo TY, Lin L, Min M, Zhao Y, He GX, Feng J, Liu WP. Primary lymphoma of breast: a clinicopathologic, immunophenotypic and prognostic study of 21 cases Zhonghua Bing Li Xue Za Zhi.Cancer 2010 May; 39(5):302-7.

16. Kuroda H, Tamaru J, Takeuchi I, Ohnisi K, Toyozumi Y, Momose S, Itoyama S. Primary diffuse large Bcell lymphoma of the breast. Breast Cancer. 2007;14(3):317-22.

17. Jennings WC, Baker RS, Murray SS, Howard CA, Parker DE, Peabody LF, Vice HM, Sheehan WW, Broughan TA. Primary breast lymphoma: the role of mastectomy and the importance of lymph node status. Ann Surg. 2007;245(5):784-9.. 